\title{
BMJ Open Association between continuity of provider-adjusted regularity of general practitioner contact and unplanned diabetes-related hospitalisation: a data linkage study in New South Wales, Australia, using the 45 and Up Study cohort
}

\author{
Rachael E Moorin, ${ }^{\oplus 1,2}$ David Youens, ${ }^{1}$ David B Preen, ${ }^{2}$ Mark Harris, \\ Cameron M Wright ${ }^{1,4}$
}

To cite: Moorin RE, Youens D, Preen DB, et al. Association between continuity of provideradjusted regularity of general practitioner contact and unplanned diabetes-related hospitalisation: a data linkage study in New South Wales, Australia, using the 45 and Up Study cohort. BMJ Open 2019;9:e027158. doi:10.1136/ bmjopen-2018-027158

- Pre-publication and additional material is published online only. To view please visit the journal online (http://dx.doi. org/10.1136/bmjopen-2018027158).

Received 9 0ctober 2018 Revised 9 April 2019 Accepted 29 April 2019

Check for updates

(C) Author(s) (or their employer(s)) 2019. Re-use permitted under CC BY-NC. No commercial re-use. See rights and permissions. Published by BMJ.

For numbered affiliations see end of article.

Correspondence to Dr Rachael E Moorin; r.moorin@curtin.edu.au

\section{ABSTRACT}

Objective To assess the association between continuity of provider-adjusted regularity of general practitioner (GP) contact and unplanned diabetes-related hospitalisation or emergency department (ED) presentation.

Design Cross-sectional study.

Setting Individual-level linked self-report and administrative health service data from New South Wales, Australia.

Participants 27409 survey respondents aged $\geq 45$ years with a prior history of diabetes and at least three GP contacts between 1 July 2009 and 30 June 2015 .

Main outcome measures Unplanned diabetes-related hospitalisations or ED presentations, associated costs and bed days.

Results Twenty-one per cent of respondents had an unplanned diabetes-related hospitalisation or ED presentation. Increasing regularity of GP contact was associated with a lower probability of hospitalisation or ED presentation (19.9\% for highest quintile, $23.5 \%$ for the lowest quintile). Conditional on having an event, there was a small decrease in the number of hospitalisations or ED presentations for the low $(-6 \%)$ and moderate regularity quintiles $(-8 \%)$, a reduction in bed days (ranging from -30 to $-44 \%$ ) and a reduction in average cost of between $-23 \%$ and $-41 \%$, all relative to the lowest quintile.

When probability of diabetes-related hospitalisation or ED presentation was included, only the inverse association with cost remained significant (mean of \$A3798 to \$A6350 less per individual, compared with the lowest regularity quintile). Importantly, continuity of provider did not significantly modify the effect of GP regularity for any outcome.

Conclusions Higher regularity of GP contact-that is more evenly dispersed, not necessarily more frequent care- - has the potential to reduce secondary healthcare costs and, conditional on having an event, the time spent in hospital, irrespective of continuity of provider. These findings argue for the advocacy of regular care, as distinct
Strengths and limitations of this study

- This study is the first to assess the impact of regularity of general practitioner (GP) contact adjusting for continuity of (GP) provider.

- This study used two-part and zero inflated negative binomial models which allowed associations to be expressed conditional on having an event and incorporating the probability of having an event.

- The assessment of the impact of provider-adjusted regularity of GP contact across subpopulations provides information that will facilitate more targeted approaches to primary care policy development.

- This study included a comprehensive range of self-reported data on health and socioeconomic status not often available in linked data studies in addition to administrative data on current and previous health service utilisation.

- This was a cross-sectional observational study over a 6-year period which limits the ability to infer causality. However, the inclusion of both health service use and outcomes prior to the study period in the modelling allowed us to partially ameliorate this limitation.

from solely continuity of provider, when designing policy and financial incentives for GP-led primary care.

\section{INTRODUCTION}

In line with other developed countries, chronic diseases predominate in Australia accounting for $66 \%$ of disease burden in 2011 and $73 \%$ of deaths in $2013 .{ }^{12}$ One strategy to address this challenge is to shift care from hospital to primary care. This is because primary care treatments are 
generally less costly ${ }^{3}$ and hospitals are not well-suited to manage chronic conditions. ${ }^{4}$ This approach is consistent with a body of literature on Ambulatory Care Sensitive Conditions; conditions for which hospitalisation may be avoidable if effective outpatient treatment is provided. ${ }^{5} \mathrm{In}$ recognition of this developing evidence base, a National Chronic Disease Strategy was devised in Australia in 2005, which promoted the integration and continuity of care. ${ }^{6}$ Similar initiatives have been introduced in the United Kingdom ${ }^{7}$ and the USA. ${ }^{8}$

Although chronic disease management in primary care has the potential to be less costly and more appropriate for patients, primary care services in Australia have historically been episodic and reactive. Such models of care are likely inadequate for patients with chronic conditions and multimorbidities. Evidence to date suggests integrated care models with a focus on chronic disease management are important for increased health system efficiency and are more responsive to consumers' needs and preferences. ${ }^{9}{ }^{10}$ In countries such as Australia, where primary care operates on a fee-for-service basis, incentive payments can be used as a mechanism to modify primary care provider behaviours in terms of how healthcare is accessed and delivered. ${ }^{11}$ Improving planned chronic disease management by general practitioners (GPs), rather than focusing on provider continuity may be appropriate in health systems with diverse funding arrangements, as there is evidence from the United Kingdom ${ }^{1213}$ and the USA ${ }^{14}$ that continuity of provider is falling.

Better linkage of care, with the GP as the central care provider, is underpinned by the theoretical notion that better continuity of care improves health and health system outcomes. GP provided care is publicly subsidised in Australia through the universal healthcare access programme, Medicare. GPs also provide a gateway to specialist and some allied health services, as a referral is required for publicly subsidised specialist or allied healthcare. The definition of 'continuity' is inconsistent and the mechanisms by which different components of continuity with a GP reduces hospitalisation are not well described. Most continuity indices focus on continuity of provider. ${ }^{15} 16$ One measure of continuity of provider is the usual provider concentration index (UPC) which is a simple measure of the proportion of visits to the same provider. ${ }^{17}$ Under most previous measures, a patient seeing a single provider on a regular, frequent basis may have a similar continuity score to a patient seeing a single provider on a very fragmented basis. For this reason, a body of work from Australia has evolved to explore the concept of 'regularity' of GP contact. ${ }^{18-20}$

Regularity refers not to the number of GP visits, but rather measures the dispersion of GP visits over time, with more even dispersion indicating better regularity. It has been shown previously that the use of financial incentives to GPs increases the regularity of primary care contact in the following year without increasing frequency of contact. ${ }^{19}$ More recently, we have reported decreased rates and costs of diabetes-related hospitalisations with increasing GP regularity. ${ }^{20}$ This finding suggests that regularity is potentially suitable as a target for policy intervention and provides some justification for the interpretation of regular primary care as indicative of proactive management of a patient or condition, as opposed to reactive or unplanned care. An important limitation of previous work has been the inability to adjust for continuity of provider when evaluating the impact of regularity. No attempt at disentangling the impact of regularity from that of provider continuity has been reported to date. Thus, despite the relevance for policy development, whether increased regularity is actually a proxy for, or a consequence of, increasing continuity of provider, or is a discrete facet of continuity of care is unknown.

The aim of this study was to assess the continuity of provider-adjusted association between regularity of GP contact and unplanned diabetes-related hospitalisations and emergency department (ED) presentations.

\section{METHODS}

\section{Study design}

This was an observational cross-sectional study using self-reported survey data linked with routinely collected unit record administrative health data from 1 July 2009 to 30 June 2015. Reporting follows the Reporting of studies Conducted using Observational Routinely collected health Data (RECORD) guidelines. ${ }^{21}$

\section{Patient and public involvement}

A consumer representative was involved in the design of the grant used to fund this research. The 45 and Up Study, which provided data for this project, maintains a repository of published research using this cohort online.

\section{Data sources}

The study used both self-reported and routinely collected administrative data linked at the person level from the 45 and Up Study. ${ }^{22}$

The Sax Institute's 45 and Up Study is a longitudinal cohort study of 266885 participants, aged 45 years and above in the state of New South Wales (NSW), Australia. Prospective participants were randomly sampled from the Australian Government Department of Human Services (DHS), formerly Medicare Australia, enrolment database and recruited from 2006 to 2009. The study methods are described in detail elsewhere. ${ }^{22}$ Briefly, participants completed a baseline health and lifestyle questionnaire and consented to follow-up and linkage to routine health databases. The overall response rate was $18 \% .^{22}$

The data sources linked and used in this study included: (1) the 45 and Up Study baseline questionnaire (https:// www.saxinstitute.org.au/our-work/45-up-study/); (2) the NSW Admitted Patient Data Collection (APDC) which provided all hospital separations in public and private hospitals in NSW (2005-2015); (3) the NSW ED Data Collection (EDDC) which provided information on all ED presentations from 2006 to 2015; (4) the Pharmaceutical 
Benefits Scheme (PBS) which provided information on subsidised prescription medicines dispensed (20052015); (5) the Medical Benefits Schedule (MBS) which provided records for all claims for medical and diagnostic services provided through Medicare, Australia's universal health insurance scheme (2005-2015) and (6) the NSW Register of Births Deaths and Marriages (RBDM) (20062015). The linkage of APDC, EDDC and RBDM to the survey data was conducted by the NSW Centre for Health Record Linkage. MBS and PBS data were linked by the Sax Institute using a unique identifier provided by the DHS. Quality assurance data on the data linkage show false-positive and false-negative rates of $<0.5$ and $<0.1 \%$, respectively. ${ }^{23}$

\section{Ascertainment of previous history of diabetes and entry into the study cohort}

This study used a cohort of 45 and Up Study participants with a history of diabetes mellitus (excluding malnutrition-related diabetes mellitus, International Statistical Classification of Diseases, 10th Revision, Australian Modification (ICD-10-AM) code E12) on or before 31 December 2009. This was ascertained via: (1) self-report from the baseline questionnaire using the question 'Has a doctor ever told you that you have diabetes?; (2) evidence of hospitalisation indicating diabetes from 2005 to 2009 using the ICD-10-AM codes E10, E11, E13, E14 ${ }^{24}$ or (3) a PBS claim indicating a dispensing from 2005 to 2009 using the following Anatomical Therapeutic Chemical codes A10A (insulins and analogues) and A10B (blood glucose lowering drugs excluding insulins).

\section{Participant inclusion and exclusion criteria}

We included participants with diabetes who had at least three GP contacts (minimum required for the UPC metric) during the study period. We excluded participants who died before 30 June 2011 to allow for a minimum of 2 years of follow-up (figure 1). Exposure (ie, patterns of GP contact) and outcomes were ascertained from 1 July 2009 to 30 June 2015 or, for participants who died during the study period, until the end of the financial year (ie, 30 June) prior to death. Over the follow-up period, a small but unknown number of participants may have moved out of NSW, where no data were collected. This would then affect collection of outcome and exposure data beyond the date in which the individual left NSW. Among those continuing to reside in NSW, follow-up for hospitalisation is considered to be $\sim 98 \%$. $^{25}$

\section{Outcomes}

Study outcomes were the number, associated bed days (for hospitalisations) and cost of unplanned diabetes-related hospitalisations or ED presentations occurring during the study follow-up period.

Hospitalisations included those classified as diabetes-related potentially preventable hospitalisations by the National Health Performance Framework (E10, E11, E13, E14) ${ }^{24}$ or where diabetes was identified as a significant risk factor by Davis $e t a l .{ }^{26}$ The ICD codes listed by Davis $e t$ $a l$ were those where diabetes was considered to increase the risk of hospitalisation for that condition. The list is freely available on table 1 of these authors' publication. ${ }^{26}$ Diabetes related, rather than all hospitalisations were chosen as an outcome because primary care for a cohort

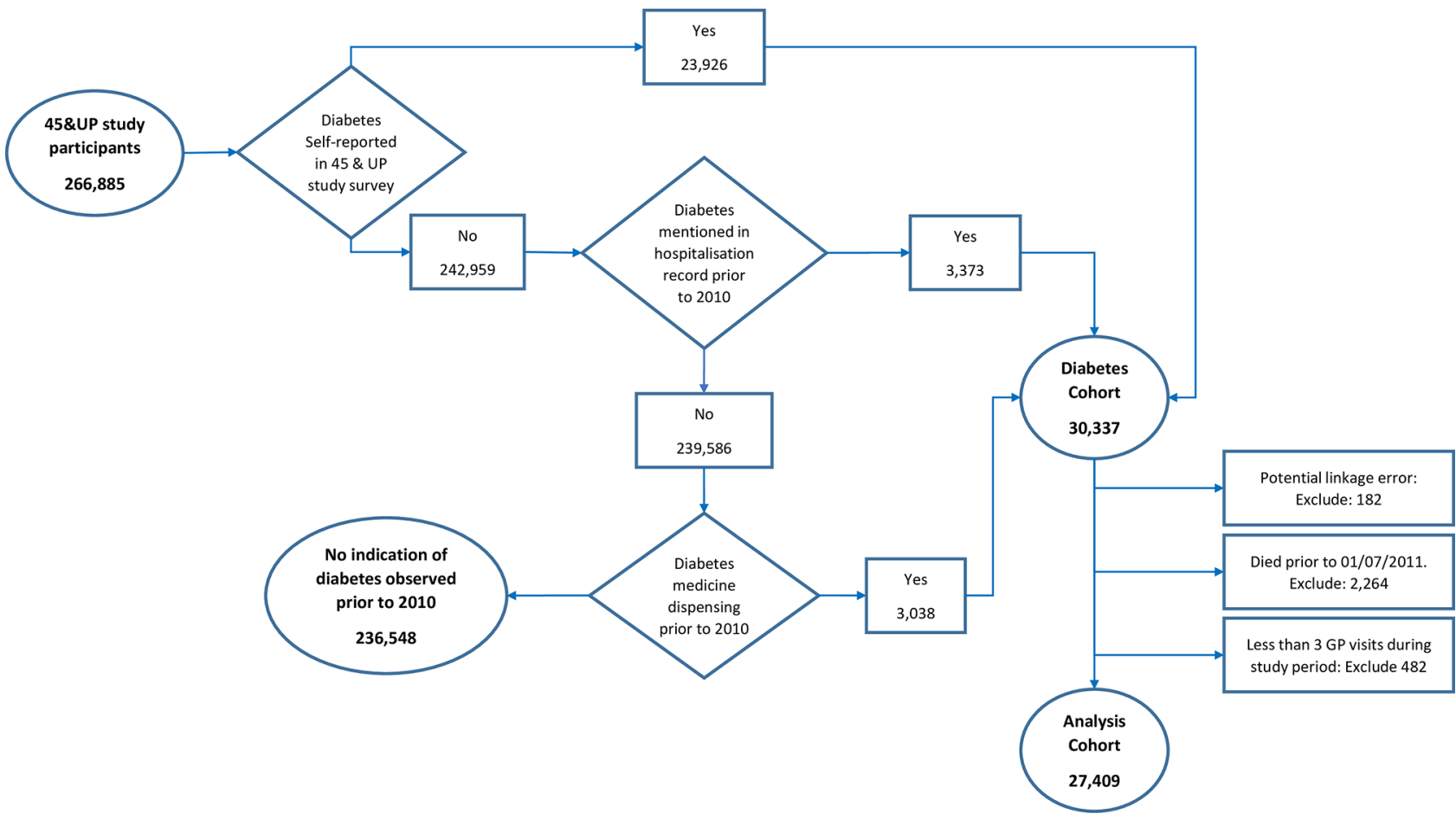

Figure 1 Cohort flow diagram. GP, general practitioner. 


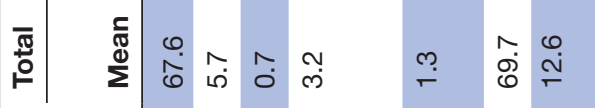

ه

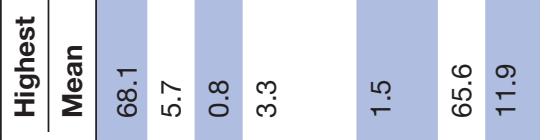

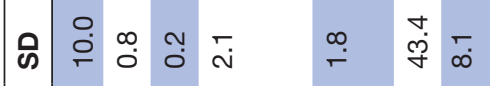

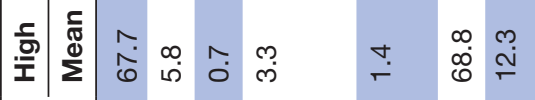

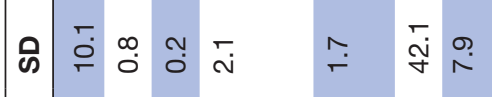

뜬

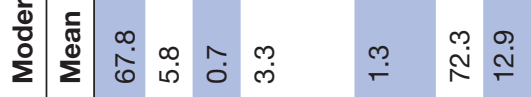

क

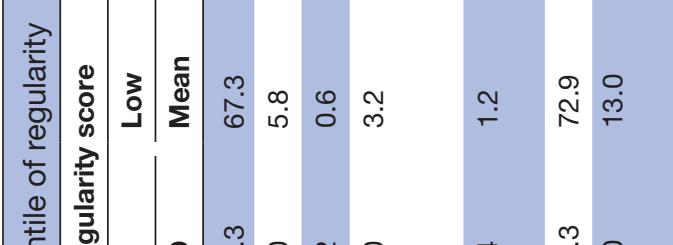

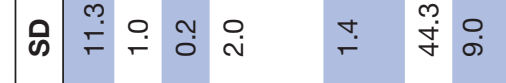

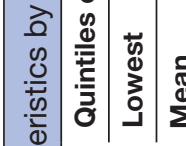

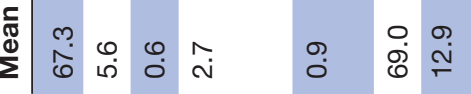

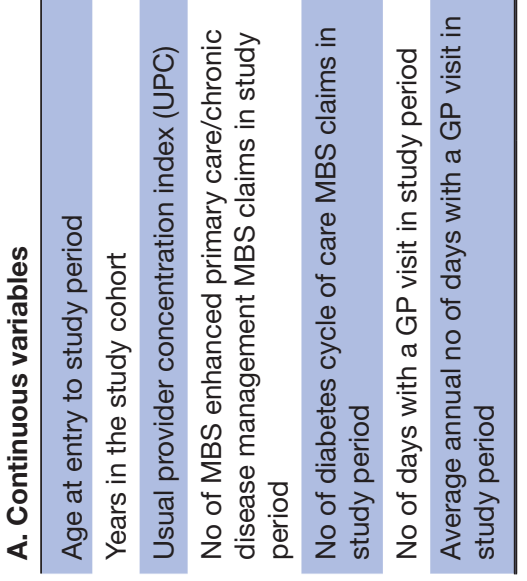

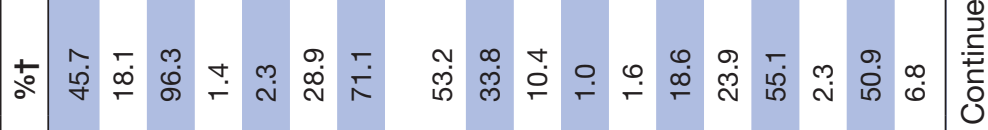

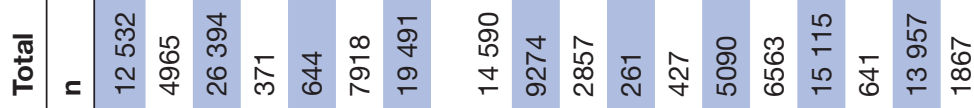

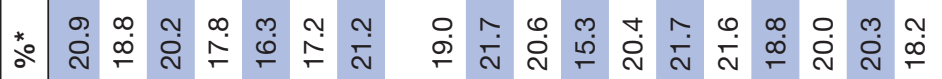

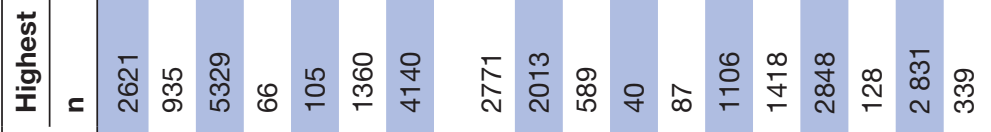

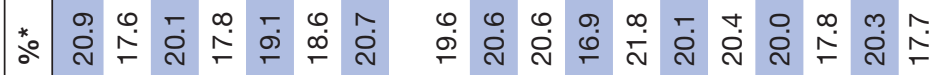

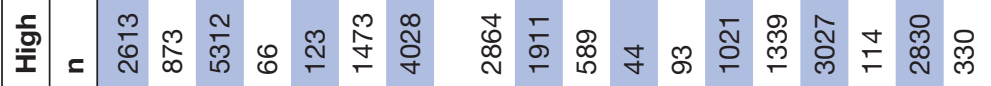

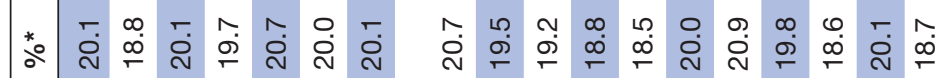
=

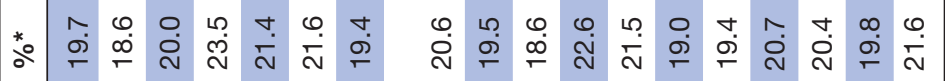

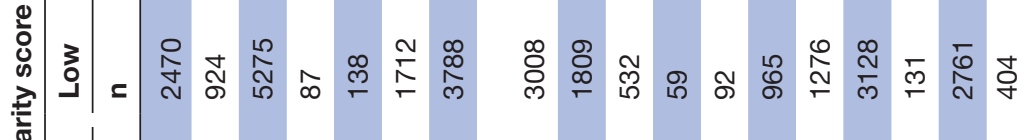

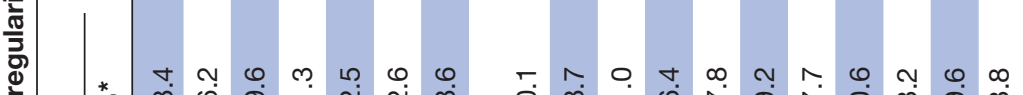

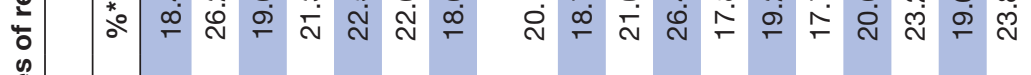
䓂|

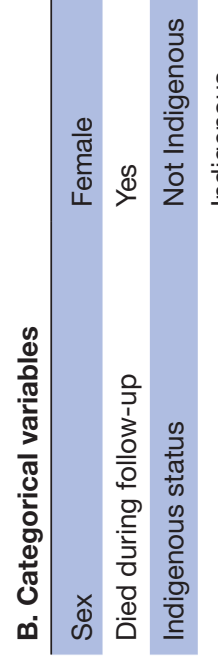

\begin{tabular}{l} 
س \\
\multirow{2}{2}{} \\
0 \\
0 \\
0 \\
\hline 1
\end{tabular}

$\stackrel{\overrightarrow{\bar{N}}}{\stackrel{9}{g}}$

흠

ฮิ

क

$\overrightarrow{\vec{\omega}}$

흥.

(1)

N

곡

운

जै

오ํ

8

흥

$\frac{0}{\otimes}$

옥

룽

$\frac{\overline{0}}{3}$

윰

호․

옥

웅

공.

N

స్

ए

足 


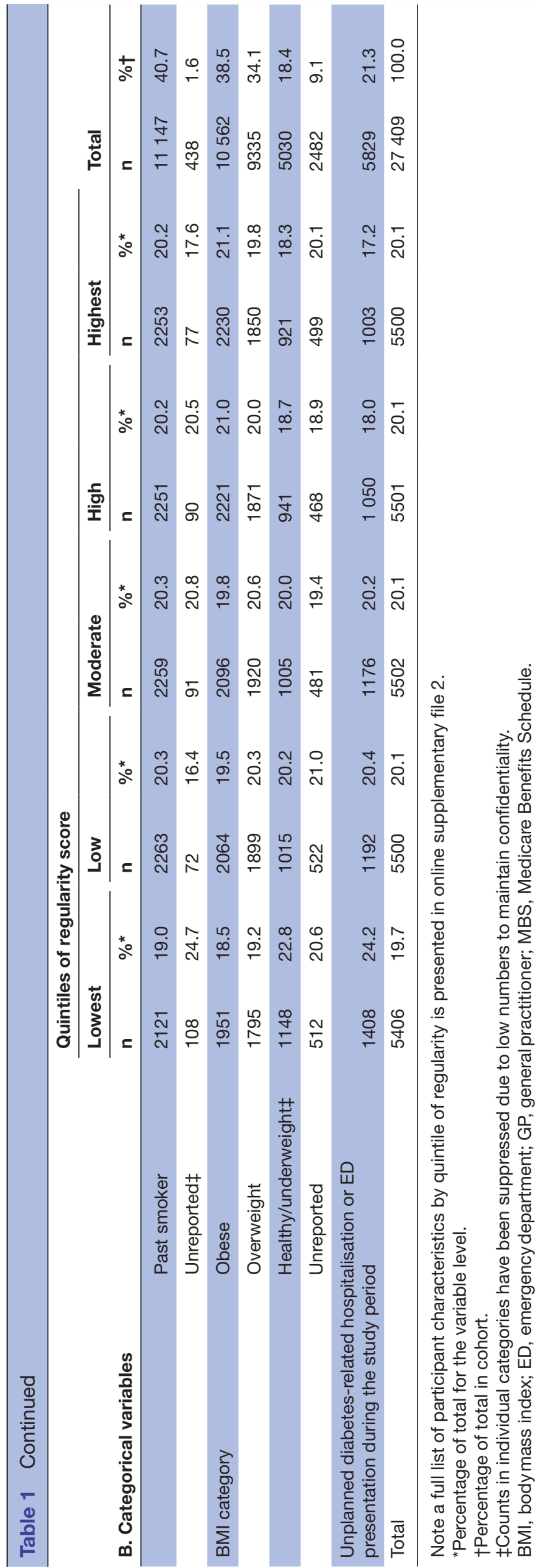

defined by having diabetes could not be reasonably expected to be associated with lower all-cause hospitalisation, and thus any association identified would be outside of the study aims. Hospitalisations were further limited to those identified as unplanned, identified using the Emergency status (urgency of admission) variable in the APDC, as these are most likely the hospitalisations that are most amenable to the potentially protective effect of better continuity of primary care. Same-day hospitalisations for routine dialysis were excluded.

Unplanned diabetes-related ED presentations were ascertained from EDDC data using either the ICD diagnosis or the Systematized Nomenclature of MedicineClinical Terms (SNOMED-CT) code, depending on availability in the data. ICD codes used to capture diabetes were those detailed above mapped to the ICD version used on the EDDC record. SNOMED-CT codes included those with a description relating to diabetes excluding where the wording included 'not related to diabetes' or relating to diabetes insipidus.

Interhospital transfers, as well as ED admissions resulting in hospitalisation, were counted as a single episode of care, with bed days calculated for each single episode of care.

Hospitalisation costs were assigned based on average public hospital Australian Refined Diagnostic-Related Group (AR-DRG) costs reported in the National Hospital Cost Data Collection round $18(2013 / 2014)$ as this was the most recent year of DRG costing in the APDC data. ${ }^{27}$ Costs were applied based on the AR-DRG code recorded for each admission. Cost of ED presentations was determined using cost weight for each urgency disposition group (UDG) using the National Efficient Price Weight Determination 2018-2019 Price Weight table. ${ }^{28}$ UDGs classify patients into 12 groups based on the type of visit, episode end status and triage code. ${ }^{29}$ UDGs were generated to reflect the UDGs reported on the National Hospital Cost Data Collection on the basis of the triage category, ${ }^{1-5}$ mode of separation and visit type reported in the EDDC data. All costs were inflated to 2017 Australian dollars using the Consumer Price Indices and included costs of nested events, where present. ${ }^{30}$

\section{Exposures}

GP contact was captured via MBS claims for 'Attendances by General Practitioners'. ${ }^{31}$ We used a modified version of the regularity index, described previously. ${ }^{19}$ Both indices use the number of days between GP visits within an ascertainment period. Whereas the original regularity score $\left(\mathrm{R}_{\mathrm{v}}\right)$ was constructed using the formula $\mathrm{R}_{\mathrm{v}}=1 /(1+$ variance (days)), the modified regularity index was calculated using the coefficient of variation $(\mathrm{Cv})$ in place of the variance. $\mathrm{Cv}$ was calculated with the formula:

$$
C v=\left(\frac{\operatorname{sd}(\text { days })}{\text { mean }(\text { days })}\right) * 100
$$

The modified index produces a unitless measure of variation, which is less correlated with frequency compared 
with previous measures. ${ }^{20}$ The modified regularity index $\left(\mathrm{R}_{\mathrm{CV}}\right)$ was constructed using the formula $\mathrm{R}_{\mathrm{Cv}}=1 /(1+\mathrm{Cv}$ (days)). This resulted in a score between 0 and 1 per individual, with 1 indicating perfectly regular contact. This score was separated into quintiles from least to most regular, using the range of scores observed in the cohort.

In addition to regularity we also used UPC, which calculates the proportion of all GP visits made to the most frequently seen GP over the study period and ranges from 0 to $1 .{ }^{1532}$ UPC was measured over the study period using deidentified provider numbers in the MBS data.

\section{Study covariates}

The 45 and Up Study questionnaire data included self-report information on key potential confounders and mediating factors including: baseline age; sex; marital status; born in Australia; Indigenous status; current housing; household income; education level; smoking history, intensity and age stopping; alcohol use; physical activity ${ }^{33}$; time spent sitting; body mass index; psychological distress $^{34}$; level of limitation reported; social support ${ }^{35}$; socioeconomic status ${ }^{36}$; and Accessibility/Remoteness Index of Australia. ${ }^{37}$ Covariate categories are provided in online supplementary file 1 .

Comorbidity was ascertained using the Multipurpose Australian Comorbidity Scoring System, ${ }^{38}$ defined as the sum of comorbidities excluding diabetes at 1 and 5 years prior to study entry. We also used the PBS data to calculate the Rx-Risk index (ie, number of condition groups excluding diabetes for which medicines were dispensed) at 1 and 5 years prior to entry to the study period. ${ }^{39}$

Health service use during and prior to the study period was captured in several ways. The number of GP and specialist physician visits 4 years before and during the study period was ascertained separately using MBS data. The frequency of contact was captured as: (1) a count of the number of days each person had a GP or specialist physician contact and (2) the SD of the annual count of days with a contact during the ascertainment period. Variables also captured if participants had a PBS records for any diabetes-related medicines, hospitalisations for dialysis, MBS diabetes cycle of care claim ${ }^{40}$ and other chronic disease related MBS-funded primary care services or glycosylated haemoglobin (HbAlc) testing during or in the 4 years prior to the study period. Finally, the number of unplanned diabetes-related hospitalisations and ED presentations in the 3 years prior to study entry was also included as a potential confounding factor.

To reduce ascertainment bias, the method (ie, self-report only, APDC only, PBS only or combinations of the three) and first year the individual who identified as having diabetes was entered into the model. In addition, a binary variable was used to determine if the participant died during the 6years of the study period. Person-time at risk of the outcome event was included in the count models.

\section{Statistical analysis}

Analyses were conducted using Stata SE Version 15 (Stata, College Station, Texas, USA). The relationship between regularity, UPC and count outcomes was evaluated in two stages. ${ }^{41}$ First, a logit model was fitted for the probability of observing the relevant outcome. Second, a negative binomial model, conditional on a positive outcome was developed. The number of days out of hospital (conditional on being alive) was used as the time at risk variable for the number of unplanned diabetes-related hospitalisations and ED presentations, while the number of years in the study cohort under observation was used as the exposure variable in the evaluation of number of bed days for unplanned diabetes-related hospitalisations. The overall relationship, accounting for the probability of having an outcome, was subsequently evaluated using zero-inflated negative binomial (ZINB) multivariable regression, which included separate components to model zero and non-zero outcomes to account for overdispersion of the data and the high proportion of person-time with no outcome. The previously defined logit and negative binomial models were used for each part of the ZINB model. For the cost outcome, a two-part model (Stata -twopm-) incorporating a logit (first part) and generalised linear model (GLM) with a gamma family and a cubed root link function for the second part were used. The GLM family was determined using the Modified Park Test. The most appropriate link function, conditional on the gamma family, was then evaluated using power links in 0.1 intervals between 1 (identity) and 0 ( $\log$ ) using Akaike Information Criterion (AIC) / Bayesian Information Criterion (BIC) and the Pearson correlation test. Once the best link function was determined, the specification of the model was evaluated using the link test.

Since the model used for cost (Stata -twopm- module) is a wrapper that facilitates computation of the combined marginal effects and adjusted predictions from the two separate models (logit and conditional GLM), comparative coefficients (ie, inflated for the probability of a zero outcome) across the outcomes do not exist. Further, the coefficients from the ZINB models cannot be directly interpreted as the impact of regularity adjusting for the probability of having an event. Rather, average marginal effects (AMEs) need to be estimated. Thus, to facilitate comparison of the impact of regularity and UPC, considering the probability of having an event in this population across outcomes, the AMEs, rather than the coefficients, were reported.

Models were built using backwards selection with retention of covariates in the parsimonious model based on statistical significance $(p \leq 0.05)$ or a change in the effect size in respect to regularity of $\geq 10 \%$. Regularity and UPC were retained in all models a priori. Competing models were evaluated using the AIC and BIC. All models incorporated robust SEs. 


\section{RESULTS}

\section{Cohort characteristics}

Nine per cent (23 926) of 45 and Up Study participants self-reported a diagnosis of diabetes (figure 1). Of those not self-reporting a diagnosis of diabetes, 3373 had a diabetes-related hospitalisation prior to 2010 and 3038 of the remaining 239586 had a diabetes medicine dispensed. The resulting diabetes cohort of 30337 was reduced to 27409 due to potential linkage error $(n=182)$, dying prior to 1 July $2011(\mathrm{n}=2264)$ or having less than three GP visits during the study period $(n=482)$.

Cohort characteristics and unadjusted outcomes are summarised in table 1, with full characteristics defined by regularity quintile and UPC category in online supplementary file 2. At entry, the diabetes cohort was $46 \%$ female. The mean age was 68 years and $1.4 \%$ of the cohort was Indigenous. The majority (71\%) were born in Australia and $73 \%$ were obese or overweight. Just over half were never smokers $(51 \%)$, had a tertiary qualification $(55 \%)$ and lived in a 'highly accessible' location (53\%). The cohort was followed for a mean of 5.7 years and averaged 13 GP visits annually. The mean reported days with a GP visit throughout the 6-year study period was higher for the lowest quintile of regularity (69) than the highest quintile of regularity (66). The number of diabetes 'cycle of care' MBS claims, HbA1c tests and chronic disease management plans were higher for the highest quintile of regularity than for the lowest. Eighteen per cent died during follow-up, with $26 \%$ of the deaths coming from the lowest quintile of regularity. Twenty-one per cent of the diabetes cohort had a diabetes-related hospitalisation or ED presentation during the study period. Forty-three per cent of participants had a UPC between 0.76 and 1.00 (online supplementary file 2). There was a positive crude association between increasing UPC and increasing regularity (table 1 ).

\section{Outcomes of conditional models}

Table 2a shows the coefficients for regularity and UPC from the multivariable logit model of unplanned diabetes-related hospitalisation or ED presentation. The full model output is shown in online supplementary file 3 . A reduction in the probability of unplanned diabetes-related hospitalisation or ED presentation was observed for each subsequent quintile compared with the lowest quintile ranging from -0.13 to -0.28 . Relative changes in the probability of an unplanned diabetes-related hospitalisation alone were similar (online supplementary file 3). Increasing UPC did not significantly change the probability of an unplanned diabetes-related hospitalisation or ED presentation $(-0.03,95 \% \mathrm{CI}-0.19$ to 0.13$)$. The adjusted probability of an unplanned diabetes-related hospitalisation or ED presentation over a 6-year period was on average 0.23 for individuals in the lowest quintile of regularity and reducing to 0.20 for those in the highest regularity quintile (figure 2A). UPC did not modify the association between quintile of regularity and the predicted probability of having a diabetes-related hospitalisation or ED presentation (online supplementary file $4 a)$.

Table 2b-d shows the model outputs for study outcomes, conditional on having one or more events over the study period; the full model outputs are shown in online supplementary file 5 . There was no consistent association between increasing regularity and the number of diabetes-related hospitalisations or ED presentations. The relative number of unplanned diabetes-related hospitalisations was significantly reduced for those with low $(-6 \%, 95 \% \mathrm{CI}-13 \%$ to $0 \%)$ and moderate $(-8 \%, 95 \%$ CI $-15 \%$ to $-2 \%$ ) compared with those with the lowest regularity. However, no significant difference existed between individuals in the highest two quintiles of regularity and their counterparts who had the lowest regularity. UPC was associated with a decrease in the relative number of diabetes-related hospitalisations or ED presentations $(-11 \%, 95 \%$ CI $-21 \%$ to $-1 \%)$. Predicted diabetes-related hospitalisations or ED presentations per 100 person-years at risk ranged from $\sim 38$ in the lowest and high quintiles of regularity to 35 in the moderate quintile of regularity (figure 2B). Increasing UPC was independently associated with a reduction in the adjusted rate of diabetes-related hospitalisations or ED presentations, but the association was not differential by quintile of regularity (online supplementary file 4b).

There was a clear negative association in the relative number of bed days, conditional on an event occurring; $-30 \%$ (CI $-38 \%$ to $-22 \%$ ) for low to $-44 \%$ (95\% CI $-53 \%$ to $-36 \%$ ) for high quintile compared with the lowest regularity quintile (table 2c). Conversely, UPC was also positively associated with the relative number of bed days $(17 \%, 95 \% \mathrm{CI} 4 \%$ to $30 \%)$. The adjusted predicted rate of bed days decreased by quintile of regularity, with a predicted 561 bed days per 100 person-years at risk for the lowest quintile of regularity, and 373 bed days for the highest quintile of regularity (figure 2C). No differential effect of quintile of regularity by UPC score was observed (online supplementary file $4 \mathrm{c}$ ).

A similar trend as described for bed days was observed for cost, with all coefficients negative relative to the lowest regularity quintile (table $2 \mathrm{~d}$ ). The reduction in cost relative to the lowest quintile was greatest in the high quintile $(-41 \%, 95 \% \mathrm{CI}-56 \%$ to $-27 \%)$, with a slightly lower reduction observed in the highest quintile $(-39 \%, 95 \%$ CI $-55 \%$ to $-23 \%)$. The adjusted predicted annual cost per 100 person-years at risk shows a range from \$A1 154557 for the lowest to $\$ A 784507$ for the high quintile of regularity. No significant association with UPC was observed ( $13 \%$ increase, $95 \%$ CI $-9 \%$ to $36 \%$ ). Online supplementary file $4 \mathrm{~d}$ shows a non-differential effect of UPC by quintile of regularity.

\section{Outcomes of unconditional models}

There was no significant difference in the number of diabetes-related hospitalisations or ED presentations across quintiles of regularity, when accounting for the probability of having an event (table 3). Similarly, the association of 


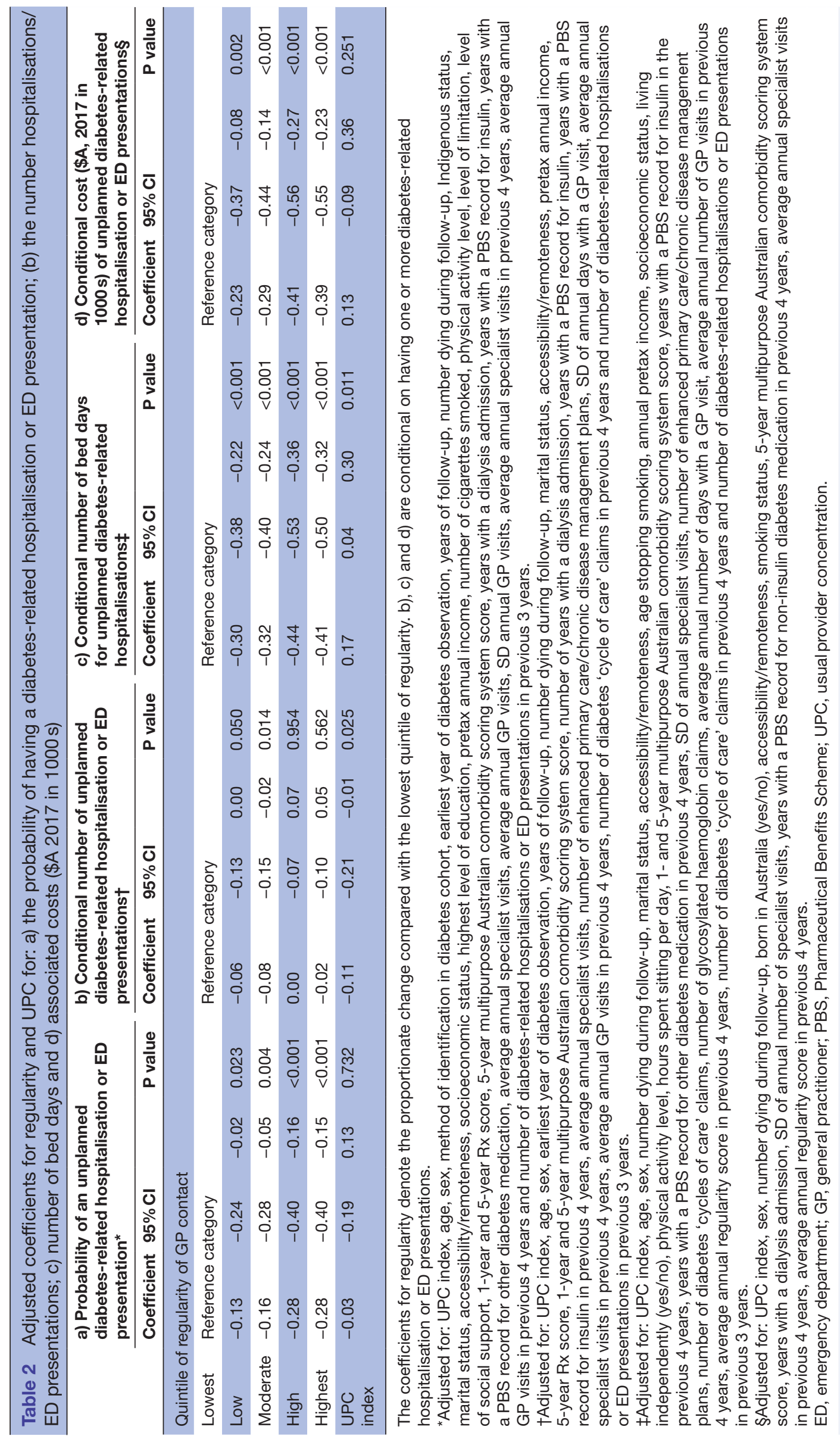


A

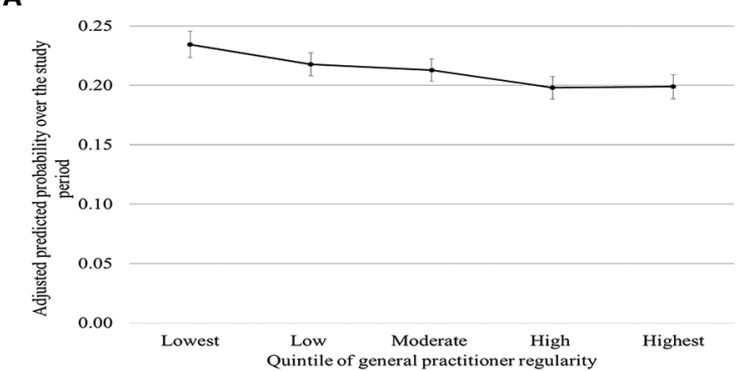

C

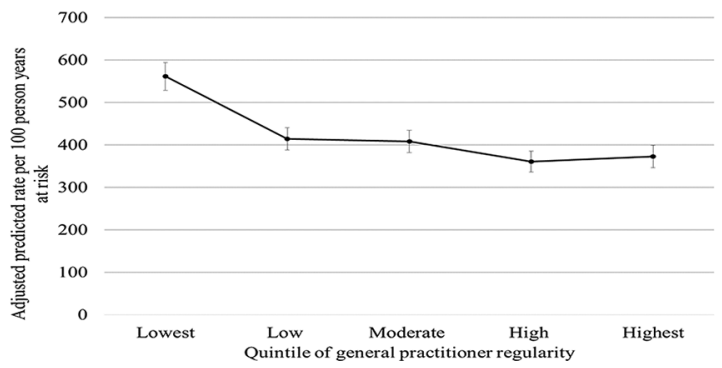

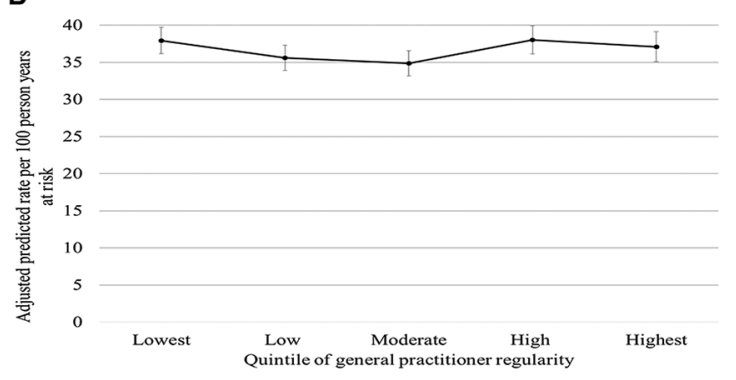

D

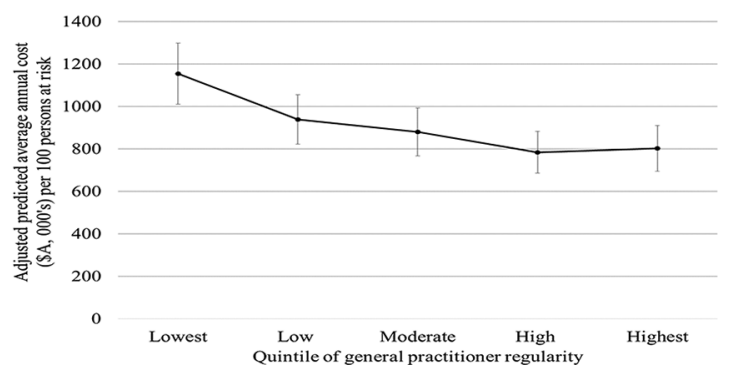

Figure 2 Adjusted ${ }^{*}$ predictions stratified by quintile of general practitioner (GP) regularity for: (A) the probability of having one or more diabetes-related hospitalisation or emergency department (ED) presentations; (B) rate of diabetes-related hospitalisation or ED presentation; (C) rate of bed days and (D) associated costs (\$A 2017 in 1000 s). (B), (C) and (D) are per 100 person-years at risk conditional on having one or more diabetes-related hospitalisation or ED presentations. *(A) Adjusted for: UPC index, age, sex, method of identification in diabetes cohort, earliest year of diabetes observation, years of follow-up, number dying during follow-up, Indigenous status, marital status, accessibility/remoteness, socioeconomic status, highest level of education, pretax annual income, number of cigarettes smoked, physical activity level, level of limitation, level of social support, 1-year and 5-year Rx score, 5-year multipurpose Australian comorbidity scoring system score, years with a dialysis admission, years with a Pharmaceutical Benefits Scheme (PBS) record for insulin, years with a PBS record for other diabetes medication, average annual specialist visits, average annual GP visits, SD annual GP visits, average annual specialist visits in previous 4 years, average annual GP visits in previous 4 years and number of diabetes-related hospitalisations or ED presentations in previous 3 years. *(B) Adjusted for: UPC index, age, sex, earliest year of diabetes observation, years of follow-up, number dying during follow-up, marital status, accessibility/remoteness, pretax annual income, 5-year Rx score, 1-year and 5-year multipurpose Australian comorbidity scoring system score, number of years with a dialysis admission, years with a PBS record for insulin, years with a PBS record for insulin in previous 4 years, average annual specialist visits, number of enhanced primary care/ chronic disease management plans, SD of annual days with a GP visit, average annual specialist visits in previous 4 years, average annual GP visits in previous 4 years, number of diabetes 'cycle of care' claims in previous 4 years and number of diabetes-related hospitalisations or ED presentations in previous 3 years. ${ }^{*}(C)$ Adjusted for: UPC index, age, sex, number dying during follow-up, marital status, accessibility/remoteness, age stopping smoking, annual pretax income, socioeconomic status, living independently (yes/no), physical activity level, hours spent sitting per day, 1-year and 5-year multipurpose Australian comorbidity scoring system score, years with a PBS record for insulin in the previous 4 years, years with a PBS record for other diabetes medication in previous 4 years, SD of annual specialist visits, number of enhanced primary care/chronic disease management plans, number of diabetes 'cycles of care' claims, number of glycosylated haemoglobin claims, average annual number of days with a GP visit, average annual number of GP visits in previous 4 years, average annual regularity score in previous 4 years, number of diabetes 'cycle of care' claims in previous 4 years and number of diabetes-related hospitalisations or ED presentations in previous 3 years. ${ }^{*}(\mathrm{D})$ Adjusted for: UPC index, sex, number dying during follow-up, born in Australia (yes/no), accessibility/remoteness, smoking status, 5-year multipurpose Australian comorbidity scoring system score, years with a dialysis admission, SD of annual number of specialist visits, years with a PBS record for non-insulin diabetes medication in previous 4 years, average annual specialist visits in previous 4 years, average annual regularity score in previous 4 years.

regularity on bed days for unplanned diabetes-related hospitalisation became non-significant. In comparison, there was a significant negative association between regularity and cost across all regularity quintiles but as described below the magnitude of the association was substantially reduced from that observed in the conditional model. The AMEs of regularity in the conditional cost model range from $-\$ A 12$ 920 (95\% CI - \$A21 2800 to - \$A4560) for the low regularity quintile to -\$A22 200 (95\% CI - \$A30 290 to - $\$$ A14 120) for the high regularity quintile. However, the AMEs for the same quintiles were reduced by approximately $70 \%$ when the probability of having a hospitalisation/ED presentation was incorporated into the model: $-\$$ A3798 (95\% CI $-\$ A 5831$ to $-\$ A 1765)$ for low regularity to $-\$ A 6530$ (95\% CI $-\$ A 8505$ to $-\$ A 4556$ ) for high regularity. The significant positive AMEs with increasing UPC with respect to bed days and cost in the conditional models disappeared in the unconditional models.

\section{DISCUSSION}

The probability of having an unplanned diabetes-related hospitalisation or ED presentation was inversely 


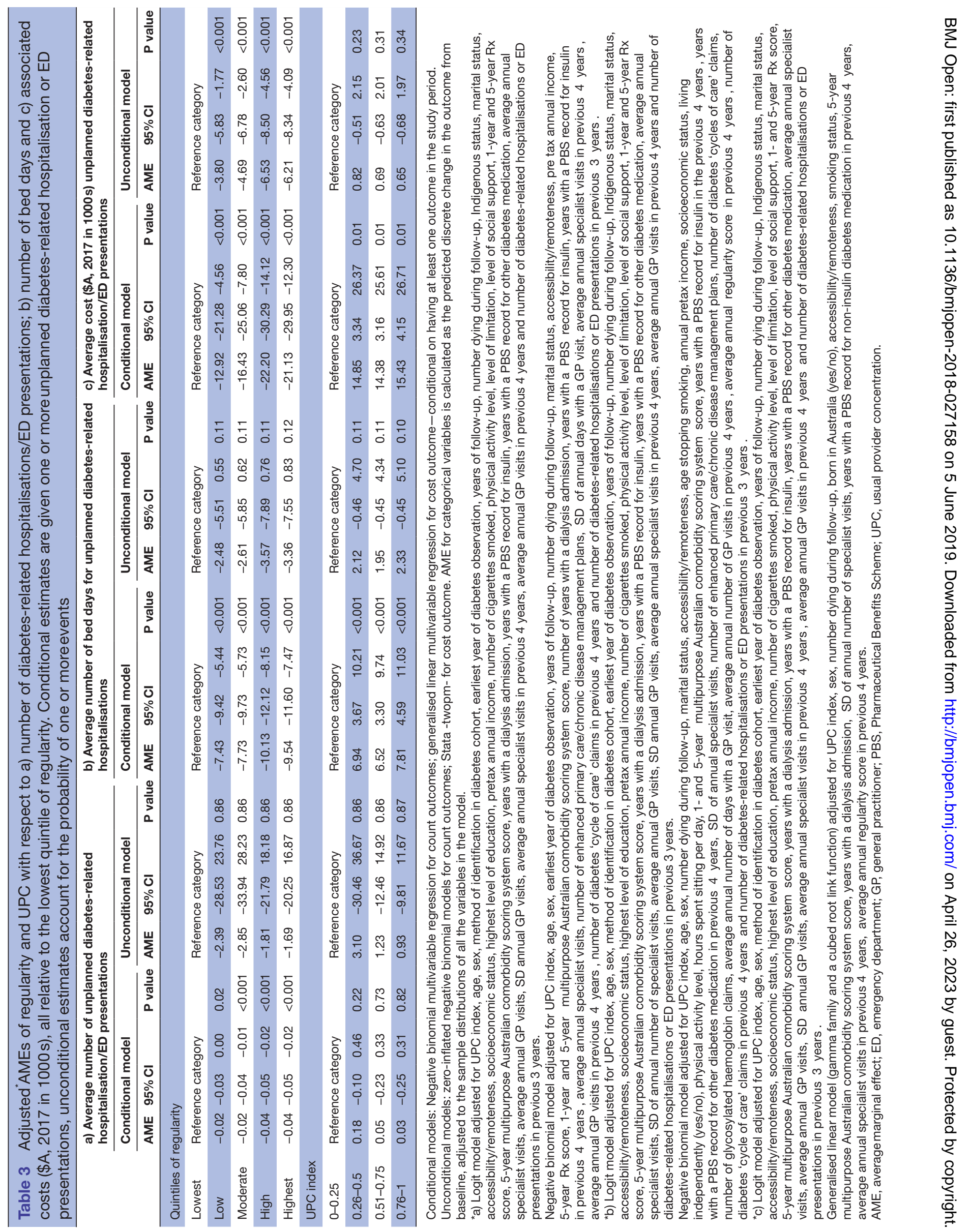


associated with regularity of GP contact, after adjusting for continuity of provider. Conditional on having an event, increasing regularity was associated with both a lower cost and reduced days in hospital. The association with cost, while somewhat attenuated, persisted when the probability of having an event was taken into account. However, the association was not significant with respect to bed days.

These findings lend support to primary care policies where proactive managed care by any GP is incentivised. In Australia, there is no formal requirement to register at a GP practice, whereas in the United Kingdom, people need to register with the National Health Service at a single GP practice. ${ }^{42}$ Campbell et $a l^{12}{ }^{43}$ report a fall in continuity of provider following implementation of the Quality Outcomes Framework (QOF), a practice-level financial incentive to improve clinical management of chronic disease in the United Kingdom. Our results suggest that this might not matter in terms of achieving a reduction in hospitalisations for people with diabetes, since the effect for regularity was larger than those of UPC when modelled together. This suggestion is corroborated by a slight fall in emergency hospitalisations for practices with more diabetic patients with moderate, as compared with poor glycaemic control, in England following implementation of the QOF. ${ }^{44}$ While there is no QOF in Australia, initiatives such as the MBS diabetes 'cycle of care' payment are similarly aimed at improving the quality of chronic disease management. ${ }^{40}$ Also using 45 and Up Study survey data, Comino et $a l^{45}$ have reported a lower adjusted rate of hospitalisation for people with diabetes for whom a diabetes cycle of care was prepared, or a GP management plan reviewed. Diabetes complications vary in both number and severity. ${ }^{46}$ The association between increasing regularity and fewer bed days (in the unconditional model) and lower cost (in both models) may indicate a lower severity of complications on average at hospital admission. Increasing regularity of GP contact will not completely negate unplanned hospitalisations, though timely intervention may allow hospital-level care to be initiated earlier. For example, a diabetic foot ulcer may still require hospitalisation for management, but avoid progression necessitating more severe and costly management. ${ }^{47}$ Ballo et $a l^{48}$ in a study assessing chronic care management for people with heart failure found an increase in hospitalisation among this group compared with controls. This was primarily driven by planned hospitalisations, for which there was a greater increase relative to unplanned. Once in place, removal of quality incentives can also change clinician behaviour, as shown recently with a decrease in documented quality measures following cessation of some of the UK's QOF elements. ${ }^{49}$

For the cost outcome, the AME was $-\$ A 6530$ for the high quintile of regularity yielding a total cohort hospitalisation cost reduction of \$A179 million. The cost-effectiveness of increasing regularity is out of scope of this study; however, this is a potential avenue for further study. Another important issue to consider is whether modelled outcome changes by regularity quintile are due to inherent differences in the people occupying each quintile, with these differences rather than any effect from GP care explaining the observed differences. There were some demographic differences across quintiles as indicated in table 1 . In our study, a wide range of covariates were accessible including sociodemographic, psychosocial, health behaviours and prior health service use, and while we cannot rule out residual confounding, the adjustment for a wide range of covariates that could plausibly affect the association reduces the risk of substantial confounding affecting interpretation.

Previous work has demonstrated an association between managed care and hospitalisations. ${ }^{1850}$ Barker et $a \tilde{P}^{51}$ have reported an association between increasing UPC and fewer ambulatory care sensitive condition hospital admissions, with a $6.2 \%$ decrease per 0.2 increase in UPC. Admissions for 22 conditions were considered by these authors making the study cohort more heterogeneous than the single-condition cohort in this study. These authors adjusted for GP frequency, but not regularity. While determination of the impact of continuity of provider was not the aim of our paper, we found a negative association between UPC (adjusted for regularity of contact) and the days spent in hospital and cost of unplanned diabetes-related hospitalisations or ED presentations, conditional on an event occurring. However, a negative association with the number of unplanned diabetes-related hospitalisations or ED presentations was not observed. Our results are, therefore, discordant with literature reporting a negative association between continuity of provider and hospitalisation. ${ }^{51-57}$ This may be explained by our simultaneous adjustment for regularity and continuity of provider. If increased continuity of provider improves regularity of contact, then studies reporting UPC without adjusting for regularity may have not differentiated between these two facets of continuity of care.

This study had several strengths. Adjustment for both regularity and continuity of provider shows that the association between regularity and study outcomes is not solely due to frequency or continuity of provider. The availability and inclusion of covariates from the 45 and Up Study and administrative data have also helped to reduce the risk of associations being due to different characteristics in each regularity quintile. Effect modification was investigated by testing the significance of an interaction term and reporting the association with regularity across the levels of UPC. Our definition of the outcome as being unplanned diabetic-related hospitalisation or ED presentations has also facilitated a deeper analysis of the impact of GP contact on a subset of diabetic-related hospitalisations that are more likely to be amenable to change by high-quality primary care. The two-part analytical methods allowed results to be presented both conditionally and unconditionally. This allowed assessment of the association between regularity and the probability of hospitalisation, the impact on additional hospitalisation for those previously hospitalised and overall for the 
population with diabetes. The latter is important because primary care policies are usually implemented across populations, and thus determining the value (impact vs cost) of the policy overall is important. Identifying if the impact differs across subpopulations is also informative, as this knowledge may facilitate more targeted approaches to policy development. There were also limitations to this study. This was a cross-sectional observational study over a 6-year period. In this study, we have reported associations, rather than to make any causal inferences. Temporal ordering of exposure followed by outcome is not given because of the cross-sectional study design. The lack of detail (eg, of length of GP consults or clinical data to indicate disease severity) in the analysis also reduces the level of detail analysed regarding these interactions. Migration outside of NSW leads to loss to follow-up. Based on mean total international and interstate migration for NSW between 2009/2010 and 2014/2015 (financial years), this proportion is at most $4.8 \%{ }^{58-60}$ However, $81.3 \%$ of these people were aged below 44 years and thus, while the total proportion of study participants affected is unknown, the impact on results is likely to be minimal $(<1 \%$ of cohort). The results of this study are not generalisable to people with diabetes below the age of 45 years. Though the response fraction in the 45 and Up Study is $18 \%$, the generalisability of results has been assessed by comparing effect measures for this cohort with those from the NSW Population Health Survey, which had a $\sim 60 \%$ response. ${ }^{61}$ These authors concluded that their 'findings show that broad ranges of exposure-outcome relationships estimated from two studies of the same population remained consistent regardless of the underlying response rate'. ${ }^{61}$

\section{CONCLUSIONS}

This is the first study to assess both regularity and continuity of provider in primary care, demonstrating an independent association between increasing GP regularity and the probability of an unplanned diabetes-related hospitalisation or ED presentation, bed days and associated costs. Based on these results, continuity of provider and regularity should be considered as distinct phenomena in future analyses, since this would aid in determining which facets of continuity are driving outcomes and aid in decisions regarding promotion of incentives that support (1) ongoing contact with the same provider (without a focus on regularity of care), (2) regular care (by any provider) or (3) regular care by the same provider. Future research should incorporate a measure of regularity, separate from provider continuity, to enable further assessment of systems-level policies to improve chronic disease management in settings with a range of funding models in primary care. The findings in this study argue for chronic disease management incentives which are not necessarily tied to a provider and invites similar adjustment in future analyses assessing the impact of GP-led primary care financial incentives. Given falling continuity of provider in some settings, levers to improve coordination of care irrespective of provider may help to reduce secondary care requirements for chronic conditions managed in primary care.

\section{Author affiliations}

${ }^{1}$ Health Systems and Health Economics, School of Public Health, Faculty of Health Sciences, Curtin University, Perth, Western Australia, Australia

${ }^{2}$ School of Population and Global Health, Faculty of Health and Medical Sciences, University of Western Australia, Perth, Western Australia, Australia

${ }^{3}$ School of Economics, Finance and Property, Curtin Business School, Curtin University, Perth, Western Australia, Australia

${ }^{4}$ School of Medicine, College of Health \& Medicine, University of Tasmania, Hobart, Tasmania, Australia

Acknowledgements This research was completed using data collected through the 45 and Up Study (www.saxinstitute.org.au). The 45 and Up Study is managed by the Sax Institute in collaboration with major partner Cancer Council NSW, and partners: the National Heart Foundation of Australia (NSW Division), NSW Ministry of Health, NSW Government Family \& Community Services - Ageing, Carers and the Disability Council NSW, and the Australian Red Cross Blood Service. We thank the many thousands of people participating in the 45 and Up Study. We also acknowledge the Commonwealth Department of Human Services for the provision of MBS and PBS data.

Contributors REM developed the study concept, conducted the analyses, interpreted results and drafted the manuscript. CMW contributed to the study design, interpreted results and drafted the manuscript. DY contributed to the study design and reviewed the draft manuscript for important intellectual content. DBP and $\mathrm{MH}$ reviewed the manuscript draft and provided comments on important intellectual content. All authors reviewed the manuscript and approved it for submission.

Funding This study was part of a project funded by the Australian National Health and Medical Research Council (APP1078345). The study funders had no role in the study design, conduct, manuscript writing or decision to submit for publication. Due to data access restrictions placed on the 45 and Up Study data, only the approved analysts (REM and DY) had access to the data for analysis, while all remaining authors, external and internal, had full access to all statistical reports and tables. REM and DY can take responsibility for the integrity of the data, while all authors can take responsibility for the accuracy of the data analysis.

Competing interests None declared.

\section{Patient consent for publication Obtained.}

Ethics approval Institutional ethics committee approval was obtained from Curtin University Human Research Ethics Committee (RD-42-14) and the NSW Population and Health Services Research Ethics Committee (HREC/17/CIPHS/37). Consent was given by all participants in the 45 and Up Study for their information to be used in approved studies, and for follow-up and data linkage. The conduct of the 45 and Up Study was approved by the University of NSW Human Research Ethics Committee.

Provenance and peer review Not commissioned; externally peer reviewed.

Data sharing statement The data that support the findings of this study are available from the relevant data custodians of the study data sets. The 45 and Up Study data were used under licence for the current study. Restrictions by the data custodians mean that the data are not publicly available or able to be provided by the authors. Researchers wishing to access the data sets used in this study should refer to the 45 and Up Study application process (www.saxinstitute.org.au/forresearchers) and the Centre for Health Record Linkage application process (www. cherel.org.au/apply-for-linked-data).

Open access This is an open access article distributed in accordance with the Creative Commons Attribution Non Commercial (CC BY-NC 4.0) license, which permits others to distribute, remix, adapt, build upon this work non-commercially, and license their derivative works on different terms, provided the original work is properly cited, appropriate credit is given, any changes made indicated, and the use is non-commercial. See: http://creativecommons.org/licenses/by-nc/4.0/.

\section{REFERENCES}

1. Australian Institute of Health and Welfare (AlHW). Australian Burden of Disease Study: Impact and causes of illness and death in Australia 
2011. Australian Burden of Disease Study series no. 3. BOD 4. Canberra, Australia: AlHW, 2016.

2. Australian Institute of Health and Welfare (AlHW). Australia's health 2016. Australia's health series no. 15. Cat. no. AUS 199. Canberra, Australia: AlHW, 2016

3. Australian Government. Australia to 2050: Future challenges. Canberra, Australia: Australian Government, 2010.

4. Armstrong BK, Gillespie JA, Leeder SR, et al. Challenges in health and health care for Australia. Med J Aust 2007;187:485-9.

5. Ansari Z, Carson N, Serraglio A, et al. The Victorian Ambulatory Care Sensitive Conditions study: reducing demand on hospital services in Victoria. Aust Health Rev 2002;25:71-7.

6. National Health Ministers' Priority Action Council. National Chronic Disease Strategy Canberra. Australia: Australian Government, Department of Health and Ageing, 2006.

7. National Health Service. Five year forward view. London, UK: National Health Service, 2014.

8. Whittington JW, Nolan K, Lewis N, et al. Pursuing the Triple Aim: The First 7 Years. Milbank Q 2015;93:263-300.

9. Ham C, Curry N. Integrated care. What is it? Does it work? What does in mean for the NHS? London, UK: The King's Fund, 2011.

10. Oliver-Baxter J, Bywood PT, Brown L. Integrated care: What policies support and influence integration in health care across New Zealand, England, Canada and the United States? PHCRIS, Adelaide: Australia Primary Health Care Research \& Information Service, 2013.

11. DoH AG. History of key MBS primary care initiatives 1999-2013 [Internet]. Canberra, Australia: Australian Government, Department of Health. 2014 http://www.health.gov.au/internet/main/publishing.nsf/ Content/mbsprimarycare-History (cited 26 September 2018).

12. Campbell SM, Kontopantelis E, Reeves D, et al. Changes in patient experiences of primary care during health service reforms in England between 2003 and 2007. Ann Fam Med 2010;8:499-506.

13. The King's Fund. Understanding pressures in general practice. London, UK: The King's Fund, 2016.

14. Ladapo J, Chokshi DA. Continuity Of Care For Chronic Conditions: Threats, Opportunities, And Policy. Health Affairs blog 2014.

15. Saultz JW. Defining and measuring interpersonal continuity of care. Ann Fam Med 2003;1:134-43.

16. Jee SH, Cabana MD. Indices for continuity of care: a systematic review of the literature. Med Care Res Rev 2006;63:158-88.

17. Breslau N, Haug MR. Service delivery structure and continuity of care: a case study of a pediatric practice in process of reorganization. J Health Soc Behav 1976;17:339-52.

18. Einarsdóttir K, Preen DB, Emery JD, et al. Regular primary care lowers hospitalisation risk and mortality in seniors with chronic respiratory diseases. J Gen Intern Med 2010;25:766-73.

19. Gibson DA, Moorin RE, Preen D, et al. Enhanced primary care improves GP service regularity in older patients without impacting on service frequency. Aust J Prim Health 2012;18:295-303.

20. Youens D, Harris M, Robinson S, et al. Regularity of contact with GPs: Measurement approaches to improve valid associations with hospitalization. Fam Pract 2019.

21. Benchimol El, Smeeth L, Guttmann A, et al. The REporting of studies Conducted using Observational Routinely-collected health Data (RECORD) statement. PLoS Med 2015;12:e1001885.

22. Banks E, Redman S, Jorm L, et al. Cohort profile: the 45 and up study. Int J Epidemiol 2008;37:941-7.

23. Korda RJ, Du W, Day C, et al. Variation in readmission and mortality following hospitalisation with a diagnosis of heart failure: prospective cohort study using linked data. BMC Health Serv Res 2017;17:220.

24. Australian Institute of Health and Welfare. National Healthcare Agreement: PI 18-Selected potentially preventable hospitalisations, 2015 [Internet]. Canberra, Australian Capital Territory, Australia, 2015 https://meteor.aihw.gov.au/content/index.phtml/itemld/559032. (cited 8 February 2019).

25. Australian Institute of Health and Welfare (AlHW). Australian hospital statistics 2011-12. Health services series no. 50. Cat. no. HSE 134. Canberra, Australia: AlHW 2013.

26. Davis WA, Knuiman MW, Hendrie D, et al. Determinants of diabetesattributable non-blood glucose-lowering medication costs in type 2 diabetes: the Fremantle Diabetes Study. Diabetes Care 2005;28:329-36.

27. Independent Hospitals Pricing Authority (IHPA). Cost weights for AR-DRG version 7.0, Round 18 (2013-14). 2016. National Hospital Cost Data Collection, Public Hospitals Cost Report, Round 18 (Financial year 2013-14) [Internet]. Sydney, NSW, Australia: IHPA, 2016. Available from. https://www.ihpa.gov.au/publications/ australian-public-hospitals-cost-report-2013-2014-round-18. (cited 26 September 2018).

28. Independent Hospitals Pricing Authority (IHPA). National Efficient Price Determination 2018-19 [Internet. Sydney, NSW, Australia: IHPA,
2018. https://www.ihpa.gov.au/publications/national-efficient-pricedetermination-2018-19. (cited 26 September 2018).

29. Independent Hospital Pricing Authority (IHPA). Urgency Related Groups and Urgency Disposition Groups [Internet. Sydney, NSW, Australia: IPHA, 2018. https://www.ihpa.gov.au/what-we-do/ urgency-related-groups-and-urgency-disposition-groups. (cited 8 June 2018)

30. Australian Bureau of Statistics (ABS). 6401.0 - Consumer Price Index, Australia, Jun 2017 [Internet. Canberra, Australia: ABS, 2017. Available from. http://www.abs.gov.au/ausstats/abs@. nsf/Previousproducts/6401.0Main\%20Features3Jun\%202017? opendocument\&tabname $=$ Summary\&prodno $=6401.0 \&$ issue $=$ Jun $\%$ 202017\&num=\&view=. (cited 8 June 2018).

31. Australian Government, Department of Health. MBS online [Internet. Canberra, Australia: Australian Government, 2018. http://www. health.gov.au/internet/mbsonline/publishing.nsf/Content/MedicareBenefits-Schedule-MBS-1. (cited 26 September 2018).

32. Dreiher J, Comaneshter DS, Rosenbluth Y, et al. The association between continuity of care in the community and health outcomes: a population-based study. Isr J Health Policy Res 2012;1:21.

33. Australian Institute of Health and Welfare (AlHW). The Active Australia Survey: a guide and manual for implementation, analysis and reporting. Canberra, Australia: AlHW, 2003.

34. Kessler RC, Andrews G, Colpe LJ, et al. Short screening scales to monitor population prevalences and trends in non-specific psychological distress. Psychol Med 2002;32:959-76.

35. Koenig HG, Westlund RE, George LK, et al. Abbreviating the Duke Social Support Index for use in chronically ill elderly individuals. Psychosomatics 1993;34:61-9.

36. Australian Bureau of Statistics (ABS). Census of population housing: Socioeconomic indexes for areas. Canberra, Australia: ABS, 2006, 2011.

37. Australian Bureau of Statistics. ABS Maps (Remoteness Structure) [Internet]. Canberra, Australia: ABS, 2006, 2011. http://stat.abs.gov. au/itt/r.jsp?ABSMaps.

38. Holman CD, Preen DB, Baynham NJ, et al. A multipurpose comorbidity scoring system performed better than the Charlson index. J Clin Epidemiol 2005;58:1006-14.

39. Pratt NL, Kerr M, Barratt JD, et al. The validity of the Rx-Risk Comorbidity Index using medicines mapped to the Anatomical Therapeutic Chemical (ATC) Classification System. BMJ Open 2018;8:e021122.

40. Australian Government, Department of Health. Medicare Benefits Schedule - Item 2517 [Internet]. Canberra, Australia: Australian Government, 2018. http://www9.health.gov.au/mbs/fullDisplay.cfm? type=item\&q=2517\&qt=item. (cited 26 September 2018).

41. Partha D, Norton EC, Manning WG. Health econometrics using Stata. College Station, Texas, United States: Stata Press, 2018.

42. National Health Service (NHS). How to register with a GP practice [Internet]. London, UK: NHS, 2016. https://www.nhs.uk/using-thenhs/nhs-services/gps/how-to-register-with-a-gp-practice/. (cited 26 September 2018).

43. Campbell SM, Reeves D, Kontopantelis E, et al. Effects of pay for performance on the quality of primary care in England. $N$ Engl J Med 2009;361:368-78.

44. Dusheiko M, Doran $\mathrm{T}$, Gravelle $\mathrm{H}$, et al. Does higher quality of diabetes management in family practice reduce unplanned hospital admissions? Health Serv Res 2011;46(1 Pt 1):27-46.

45. Comino EJ, Islam MF, Tran DT, et al. Association of processes of primary care and hospitalisation for people with diabetes: A record linkage study. Diabetes Res Clin Pract 2015;108:296-305.

46. Young BA, Lin E, Von Korff M, et al. Diabetes complications severity index and risk of mortality, hospitalization, and healthcare utilization. Am J Manag Care 2008;14:15-23.

47. Mishra SC, Chhatbar KC, Kashikar A, et al. Diabetic foot. BMJ 2017;359:j5064.

48. Ballo P, Profili F, Policardo L, et al. Opposite trends in hospitalization and mortality after implementation of a chronic care model-based regional program for the management of patients with heart failure in primary care. BMC Health Serv Res 2018;18:388.

49. Minchin M, Roland M, Richardson J, et al. Quality of Care in the United Kingdom after Removal of Financial Incentives. N Engl J Med 2018;379:948-57.

50. Einarsdóttir K, Preen DB, Emery JD, et al. Regular primary care plays a significant role in secondary prevention of ischemic heart disease in a Western Australian cohort. J Gen Intern Med 2011;26:1092-7.

51. Barker I, Steventon A, Deeny SR. Association between continuity of care in general practice and hospital admissions for ambulatory care sensitive conditions: cross sectional study of routinely collected, person level data. BMJ 2017;356:j84. 
52. Christakis DA, Wright JA, Koepsell TD, et al. Is greater continuity of care associated with less emergency department utilization? Pediatrics 1999;103(4 Pt 1):738-42.

53. Gill JM, Mainous AG. The role of provider continuity in preventing hospitalizations. Arch Fam Med 1998;7:352-7.

54. Wasson JH, Sauvigne AE, Mogielnicki RP, et al. Continuity of outpatient medical care in elderly men. A randomized trial. JAMA 1984;252:2413-7.

55. Cheng S-H, Chen C-C, Hou Y-F. A Longitudinal Examination of Continuity of Care and Avoidable Hospitalization. Arch Intern Med 2010;170:1671-7.

56. Christakis DA, Mell L, Koepsell TD, et al. Association of lower continuity of care with greater risk of emergency department use and hospitalization in children. Pediatrics 2001;107:524-9.

57. Cho KH, Lee SG, Jun B, et al. Effects of continuity of care on hospital admission in patients with type 2 diabetes: analysis of nationwide insurance data. BMC Health Serv Res 2015;15:107.

58. Australian Bureau of Statistics. 3101.0 - Australian Demographic Statistics, Jun 2018. TABLE 4. Estimated Resident Population, States and Territories (Number) [Internet]. Canberra, Australian Capital Territory, Australia: Australian Bureau of Statistics, 2018. Available from. https://www.abs.gov.au/AUSSTATS/abs@.nsf/DetailsPage/ 3101.0Jun\%202018?OpenDocument. (cited 12 February 2019).

59. Australian Bureau of Statistics. Interstate migration: Arrivals, departures and net, State/territory, Age and sex - Financial years, 1996-97 onwards [Internet]. Canberra, Australian Capital Territory, Australia: Australian Bureau of Statistics, 2019. http://stat.data.abs. gov.au/Index.aspx?DatasetCode=INTERSTATEMIGRATION_FY. (cited 12 February 2019).

60. Australian Bureau of Statistics. Net overseas migration: Arrivals, departures and net, State/territory, Age and sex - Financial years, 2004-05 onwards [Internet. Canberra, Australian Capital Territory, Australia: Australian Bureau of Statistics, 2019. http://stat.data.abs. gov.au/Index.aspx?DatasetCode=NETOVERSEASMIGRATION_FY. (cited 12 February 2019).

61. Mealing NM, Banks E, Jorm LR, et al. Investigation of relative risk estimates from studies of the same population with contrasting response rates and designs. BMC Med Res Methodol 2010;10:26. 